\title{
CD146 detection with real-time total internal reflection imaging ellipsometry
}

\author{
Li Liu ${ }^{1,3}$,Yu Niu ${ }^{1,3}$,YongHong Meng ${ }^{1}$,She Chen $^{1}$, XiYun Yan ${ }^{2}$, Gang Jin $^{1 *}$ \\ ${ }^{1}$ Institute of Mechanics, Chinese Academy of Sciences, Beijing 100190, China \\ ${ }^{2}$ Institute of Biophysics, Chinese Academy of Sciences, Beijing 100101, China \\ ${ }^{3}$ Graduate School, Chinese Academy of Sciences, Beijing 100049, China \\ *Corresponding author: E-mail: gajin@imech.ac.cn; Tel./Fax.: +86-10-82544138
}

\begin{abstract}
Biosensor with the total internal reflection imaging ellipsometry (TIRIE) uses an evanescent wave as optical probe to monitor bio-molecular interaction with a high sensitivity due to its property of phase sensitive. Here, the biosensor is applied for a quantitative detection of CD146 with concentrations of 0.1 to 100 $\mathrm{ng} / \mathrm{mL}$ in order to realize a high sensitive quantitative detection. Moreover, the regression curve between the signal of biosensor $(y)$ and CD146 concentration $(x=$ $\ln C+2.4)$ is deduced as a linear $y=1.0544 x+0.7839$.
\end{abstract}

Keywords-TIRIE biosensor, CD146 detection

\section{INTRODUCTION}

Adhesion molecule CD146 (100-130kDa) belongs to the immunoglobulin super family and it is originally identified as a biomarker for melanoma [1] . Recently, CD146 is found as novel target molecule on endothelial cell and involved in tumor angiogenesis [2] . Also CD146 is considered as critical molecule in cell invasion $[3 ; 4 ; 5]$ and antiCD146 antibody could inhibit tumor metastasis and angiogenesis through its down regulation of NFk [6;7]. In this paper we attempt to detect the CD146 molecule in positive serum with the TIRIE biosensor.

TIRIE is imaging ellipsometry performed in the total internal reflection mode which is introduced previously [8] .The biosensor with TIRIE using evanescent wave as optical probe to observe bio-molecular interaction has high sensitivity due to its phase sensitive [9], otherwise avoids the solution disturbance and transparency influence. It is a powerful tool for the visualization and analysis of biomolecular mono-layers. It can be operated in real time mode for the bio-molecular interaction process detection. Its properties are fast sampling for a large field of view, nondisturbance, qualitative and quantitative detection with label free and low reagent and specimen consumption. Its multichannel micro-reactor has functions of the solution delivery, the ligand immobilization, the surface blocking, and a high throughput detection.

\section{MATERIALS AND METHODS}

\section{A. Materials}

The SF10 glass slides were purchased from Changchun institute of Optics, Fine Mechanics and Physics, Chinese Academy of Sciences (China). The 11-mercaptoundecanoic acid (MUA) was purchased from Sigma (USA).1-(3Dimethylaminopropyl)-3-ethylcarbodiimide hydrochloride (EDC) and N- hydroxy-droxysuccinimide (NHS) were purchased from ACROS. All chemicals for preparing Phosphate-buffered Saline (PBS, $10 \mathrm{mM}$ phosphate, $0.1 \mathrm{M} \mathrm{NaCl}$, pH 7.4) and PBS solution with $1 \%$ Tween 20 (PBST) were purchased from sigma. Deionized water (resistivity 18.3 $\mathrm{M} \Omega \mathrm{cm}$ ) was produced by ion exchange demineralization, followed by passing through a Milli-Q plus system from Millipore (Millipore, Bedford, MA). Mouse monoclonal antibody to CD146 AA1 was provided by institute of Biophysics of Chinese Academy of Sciences. All human sera were supplied from Anzhen Hospital (China).

\section{B. Substrate}

The SF10 glass slide was used as substrate and prepared by the evaporation of $2 \mathrm{~nm}$ of chromium on surface then added by the evaporation of $30 \mathrm{~nm}$ of gold. The gold surface was immersed into a $1 \mathrm{mM}$ MUA- ethanol solution for at least $18 \mathrm{Hs}$, followed by a thorough rinsing with both ethanol and deionized water. The MUA mono-layer with carboxyl group was self-assembled on the gold surface.

\section{TIRIE setup}

The schematic of the biosensor is shown in Figure 1. The TIRIE biosensor system is based on imaging ellipsometer under the total internal reflection condition equipped with a 


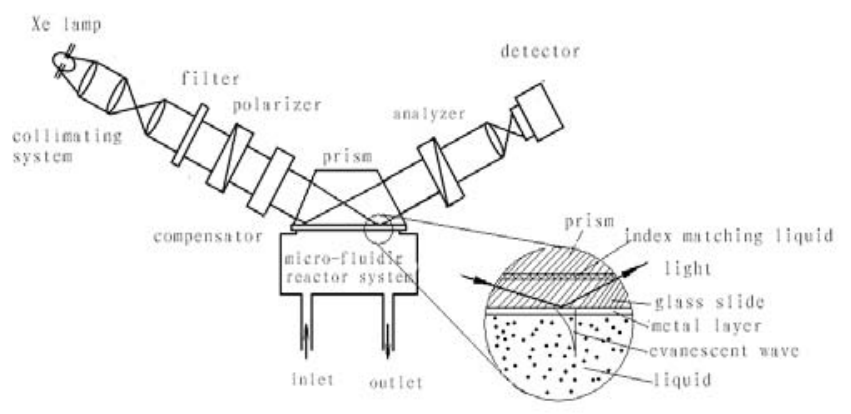

Figure 1.Schematic of the TIRIE biosensor. The zoom shows the details of the environment around the protein chip.

coupling prism and micro-fluidic reactor system. The principle of the TIRIE is briefly described here, and more details can be found elsewhere $[10 ; 11]$. Light beam with wavelength $633 \mathrm{~nm}$ from light source goes through the polarizer and the compensator and then passes a $59^{\circ}$ prism. The substrate equipped with the protein micro-array on chip mounted on the flow cell of the micro-fluidic reactor system contacts the prism bottom. For the prism and the glass slides coupling, an index matching liquid is used between them (refractive index $n=1.73$ ). After reflection from the bio-chip surface the light beam goes through the analyzer and then focused on a sensing area of the charge coupled device (CCD) camera. The video signal corresponding to the biomolecular mass surface concentration distribution was captured, digitized and stored in gray-scale format (8bits $0-256$ grayscale) in a computer.

\section{RESULTS AND DISCUSSION}

The goal of the experiment is to detect the target CD146 molecule with low concentrations in normal serum and obtain a regression curve of biosensor signal versus the concentration of target molecule. The ligand of target molecule is AA1 the monoclonal antibody of CD146. The immobilization condition of the ligand is determined by an optimization result of the concentration (mouse ascites diluted 200 times with PBS buffer) and the immobilization time is $10 \mathrm{~min}$. In our experiment, the concentration of CD146 in sera is $0.1,1,10,20,40,100 \mathrm{ng} / \mathrm{mL}$, respectively. All results of CD146 real-time detection processes are shown in Figure 2. The zoom in Figure 2 is the analysis of regression curve of Region 7 and Region 8 which show the dynamic process of CD146 with different concentrations reacting with its specific antibody on surface. Curve markers from $\mathrm{g}$ to a correspond to different concentrations of CD146 from low to high. The signal in grayscale before and after the interaction is shown in Table 1 . The data in the region 8 is averaged over the last $300 \mathrm{~S}$ indicated in the Figure 2 .

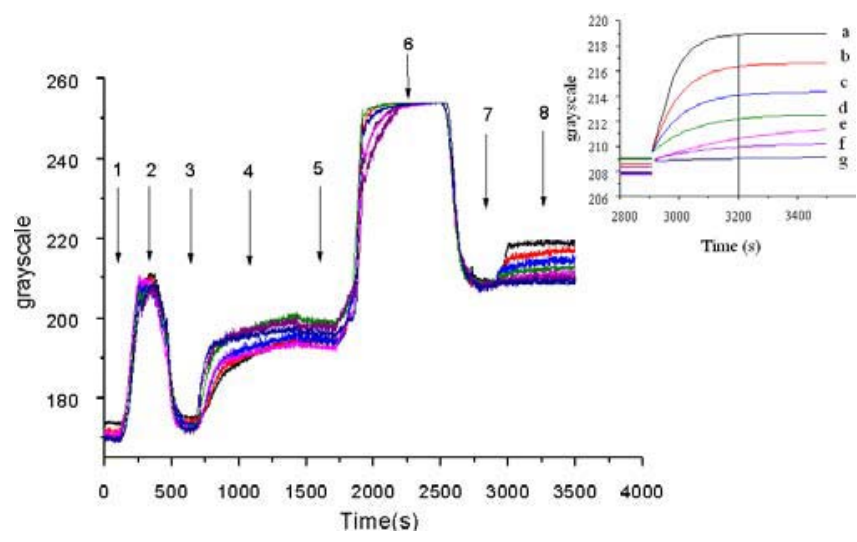

Figure 2. the real-time curves of the dynamic process for the CD146 binding with its antibody AA1 in various concentrations. The 1st region was the baseline. The 2 nd region was corresponding to the NHS-EDC activation of carboxyl group assembled on the gold substrate; 3rd -PBS rising; $4^{\text {th }}$ - AA1(1:200) immobilization on gold-coated substrate surface; $5^{\text {th }}-$ PBS rinsing, $6^{\text {th }}-$ blocking, $7^{\text {th }}-$ PBS rinsing and $8^{\text {th }}-C D 146$ binding with its antibody. Curve a-f was related to the several CD146 concentrations of $100,40,20,10,1,0.1 \mathrm{ng} / \mathrm{ml}$. The curve $\mathrm{g}$ was the reference.

Table 1: the surface concentration in grayscale before and after CD146 binding with its antibody

\begin{tabular}{|c|c|c|c|}
\hline $\begin{array}{c}\text { Concentration(C) } \\
(\mathrm{ng} / \mathrm{ml})\end{array}$ & $7^{\text {th }}$ Region & $8^{\text {th }}$ Region & $\begin{array}{l}\text { Difference be- } \\
\text { tween ligand and } \\
\text { interaction region }\end{array}$ \\
\hline 100 & $209.1 \pm 0.7$ & $218.4 \pm 0.4$ & $9.3 \pm 1.1$ \\
\hline 40 & $208.8 \pm 0.4$ & $216.5 \pm 0.5$ & $7.7 \pm 0.9$ \\
\hline 20 & $208.0 \pm 0.5$ & $214.4 \pm 0.6$ & $6.4 \pm 1.1$ \\
\hline 10 & $209.0 \pm 0.4$ & $212.4 \pm 0.4$ & $3.4 \pm 0.8$ \\
\hline 1 & $208.1 \pm 0.5$ & $211.6 \pm 0.5$ & $3.5 \pm 1.0$ \\
\hline 0.1 & $208.4 \pm 0.3$ & $209.9 \pm 0.3$ & $1.5 \pm 0.6$ \\
\hline 0 & $208.2 \pm 0.2$ & $209.1 \pm 0.3$ & $0.9 \pm 0.5$ \\
\hline
\end{tabular}

Though the signal of the biosensor is quite low at the concentration $0.1 \mathrm{ng} / \mathrm{ml}$ but significant compared with the reference. It confirms that the sensitivity of the biosensor reaches the order of $\mathrm{ng} / \mathrm{mL}$. For quantification, the regression curve is established between the signal of biosensor and the concentration of CD146(C). The relationship between the signal in grayscale difference (y) and the concentration appears a logarithm formulation $(\mathrm{x}=\ln \mathrm{C}+2.4)$ especially in lower concentration showed in Figure 3 . The signal variation is proportional to the concentration as expected in the regressive fitting curve and the relationship between the signal in grayscale difference(y) and concentration of CD146 ( $\mathrm{x})$ is $\mathrm{y}=1.0544 \mathrm{x}+0.7839$.

The signal corresponds to $10 \mathrm{ng} / \mathrm{mL}$ appears abnormal in regression curve. The signals of $10 \mathrm{ng} / \mathrm{mL}$ and $20 \mathrm{ng} / \mathrm{mL}$ 
are so quite close that it's hard to distinguish. Maybe it's impacted by the ununiformity among independent channels or the stochastic noise, so that the improvement of the uniformity among independent channels and the ratio of signal and ratio is required. The work for the improvement is on the way, and some improved results could be foreseen.

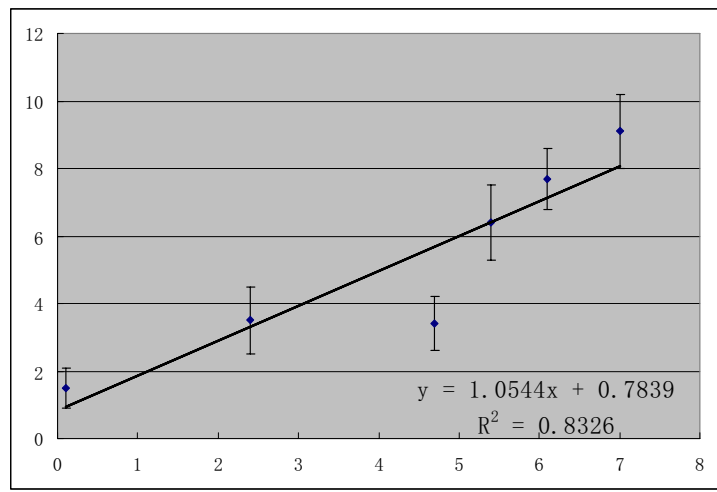

Figure 3. the regressive fitting curve between the signal in grayscale difference(y) in Table 3 and the concentration (C) of CD146 (x).. Here, the relationship between $\mathrm{x}$ and the concentration of CD146 is $\mathrm{x}=\ln \mathrm{C}+2.4$.

\section{Conclusion}

CD146 with concentration of $0.1-100 \mathrm{ng} / \mathrm{ml}$ range in serum has been detected with the TIRIE biosensor dynamically and quantitatively. The concentration of the detected sample is lower than the standard sample $243 \mathrm{ng} / \mathrm{mL}$ which confirmed the biosensor could be applied in CD146 of low concentration detection. A linear relationship $y=1.0544 x+0.7839$ between the biosensor signal $(y)$ and the concentration of CD146 with logarithm formulation $(\mathrm{x}=\ln \mathrm{C}+2.4)$ has been obtained by regression curve which would be used as a reference of calibration for further CD146 detection. The total internal reflection biosensor shows a potential for clinic application.

\section{REFERENCES}

[1]Johnson J P, Rothbacher USers C (1993) The progression associated antigen muc18 - a unique member of the immunoglobulin supergene family. Melanoma Res. 3:337-340.

[2]Bu P C, Zhuang J, Feng J et al. (2007) Visualization of cd146 dimerization and its regulation in living cells. Biochim. Biophys. Acta-Mol. Cell Res. 1773:513-520.

[3]Lehmann J M, Holzmann B, Breitbart E W et al. (1987) Discrimination between benign and malignant-cells of melanocytic lineage by 2 novel antigens, a glycoprotein with a molecular-weight of 113,000 and a protein with a molecular-weight of 76,000. Cancer Res. 47:841845 .

[4]Lehmann J M, Riethmuller GJohnson J P (1989) Muc18, a marker of tumor progression in human-melanoma, shows sequence similarity to the neural cell-adhesion molecules of the immunoglobulin superfamily. Proc. Natl. Acad. Sci. U. S. A. 86:9891-9895.

[5] Kang Y Y, Wang F C, Feng J et al. (2006) Knockdown of cd146 reduces the migration and proliferation of human endothelial cells. Cell Res. 16:313-318

[6]Yan X Y, Lin Y, Yang D L et al. (2003) A novel anti-cd146 monoclonal antibody, aa98, inhibits angiogenesis and tumor growth. Blood 102:184-191

[7]Bu P C, Gao L Z, Zhuang J et al. (2006) Anti-cd146 monoclonal antibody aa98 inhibits angiogenesis via suppression of nuclear factorkappa $b$ activation. Mol. Cancer Ther. 5:2872-2878.

[8]Chen Y YGang J (2006) Biosensor based on total internal reflection imaging ellipsometry. The Ninth World Congress on Biosensors.Canada: Biosensors \& Bioelectronics, 219 :

[9]G. Jin R J, H.Arwin (1996) Imaging ellipsometry revisited:Developments for visualization of thin transparent layers on silicon substrates. Rev.Sci.Instrum 67:2930-2936.

[10]Arwin H, Poksinski MJohansen K (2004) Total internal reflection ellipsometry: Principles and applications. Appl. Optics 43:3028-3036. [11]Wang G L, Arwin HJansson R (2004) Optimization of off-null ellipsometry in sensor applications. Appl. Optics 43:2000-2005. 